\title{
Oscillation Ring Testing Methodology of TSVs in 3D Stacked ICs
}

\author{
Shadi MS Harb \\ Intel Corporation \\ Hillsboro OR 97124 \\ USA \\ shadiharb@ieee.org
}

\author{
William Eisenstadt \\ Electrical and Computer Engineering Dept. \\ University of Florida, Gainesville, FL 32611 \\ USA \\ wre@tec.ufl.edu
}

\begin{abstract}
This paper presents on-chip self-testing circuits to detect faulty Through Silicon Vias (TSVs) in 3D ICs technology. Different testing schemes based on an oscillation ring testing methodology are proposed to detect TSVs faults such as stuck-at, open, slope and delay degradation, and severe crosstalk TSVs coupling. A parallel ring-based oscillator test structure is proposed and simulated based on a high performance fully tunable electrical circuit pi-model where a single and coupled TSVs with groundsignal-ground (GSG) and ground-signal-signal-ground (GSSG) 3D vias configurations are used as a test vehicle for 3D interconnect characterization and test. Simulation results are presented using the Keysight/Agilent Advance Design System (ADS) and a standard $0.25 \mu \mathrm{m}$ CMOS process.
\end{abstract}

Keywords- Interconnect, Crosstalk, 3D Technology, TSVs, Test, Stuck-at, Oscillation Ring, pi-model, ADS.

\section{INTRODUCTION}

$\mathrm{W}$ th deep sub-micron silicon processing technology, it becomes more difficult for integrated circuits to achieve higher integration through the scaling down of the transistor feature size. 3D ICs technology stacks multiple dies together and connects them using Through-Silicon Vias (TSVs), which become a promising technology to provide a low cost and high efficient way to increase integration for modern systems. However, there are some problems associated with this technology. One of the most important issues is the compound yield loss due to die stacking and TSV failures, which might occur either in the fabrication of the TSV themselves, in the bonding of the TSVs to the next layer, or during the life time of the 3D stack. Thus, to guarantee the stacking yield, the interconnections (TSVs) reliability must be tested. Common TSV defects in this emerging technology can be caused by TSV shorts, opens, delay faults, crosstalk induced-delay faults and glitches. To insure a low probability of 3D IC failure, integrating embedded test structures in 3D stacked ICs becomes a more attractive solution to avoid traditional probe testing, which becomes an infeasible approach to validate inaccessible nodes after stacking. The embedded test circuits can be integrated during the fabrication process to test a group of TSVs or each TSV individually for different possible defects in the die before stacking. In addition, they can be used to characterize the TSV's signal integrity effects and the impact of TSVs on the $3 \mathrm{D}$ circuit performance after fabrication. The results of these test circuits can then be read out off-chip for post processing. To extend the ring oscillator concept, which is considered as a fundamental circuit in literature used to evaluate the performance of various IC technologies, an oscillation ring (OR) 3D test methodology with different circuits testing schemes are proposed in this paper to detect TSVs failure induced by TSVs shorts, opens, delay faults and crosstalk coupling.

This paper is organized as follows: a high performance TSV electrical pi-model, which can be used as DUT for 3D TSV testing, is discussed in Section II. Section III proposes ringbased oscillation testing schemes to detect TSVs failures and their induced 3D performance degradation. Section IV concludes the paper.

\section{AN EQuivalent ElECTRICAL CIRCUIT For TSVS}

To test and study the effects of TSVs on 3D circuit performance, a high frequency electrical pi-model, commonly used in literature [1-3], is applied for TSV test and characterization, which is extracted from physical configurations of a common TSV structure. Ground-signalground (GSG) and ground-signal-signal-ground (GSSG) vias configurations are used to test single and coupled TSVs for different $3 \mathrm{D}$ routing topologies. By leveraging these fully parametric TSV models, different TSV designs can be explored to study the impact of TSVs on the 3D performance. In addition, changing the model parameters gives more insight about how these parameters such as induced resistance and capacitance of TSVs affect the 3D circuit. Figure 1(a) shows a schematic drawing of the high frequency equivalent circuit model of a GSG via configuration [4].

The electrical model of the TSV is expressed as an inductor (Lvia) and a resistor (Rvia), while the capacitive coupling between the vias is modeled as coupling capacitors (Cvia ox, Cox, and Csil). The parameter Cvia_ox denotes the capacitance of the thin oxide layer surrounding the via barrel, and Cox denotes the capacitance of the oxide layer on the silicon surface and the fringing field between the vias. The capacitance of the silicon substrate is denoted by Csil, and the loss property of the silicon substrate between the signal via and the ground via is denoted by Gsil. The model parameters (Rvia $=0.22 \Omega$, Lvia $=3$ $\mathrm{pH}$, Cvia ox $=1 \mathrm{PF}, \mathrm{Cox}=5 \mathrm{fF}, \mathrm{Csil}=4 \mathrm{fF}, \mathrm{Gsil}=2.92 \mathrm{~m} / \Omega$ ) are 
chosen from [3] as default values for the proposed testing applications. It is worth mentioning that the Rvia and Cvia_ox seem to be the main parameters to consider in order to model the impact of TSVs on 3D circuits. Figure 1(b) shows also the pi electrical model for a GSSG structure. It is assumed that the two outer TSVs are grounded and the other two inner ones are signal TSVs.

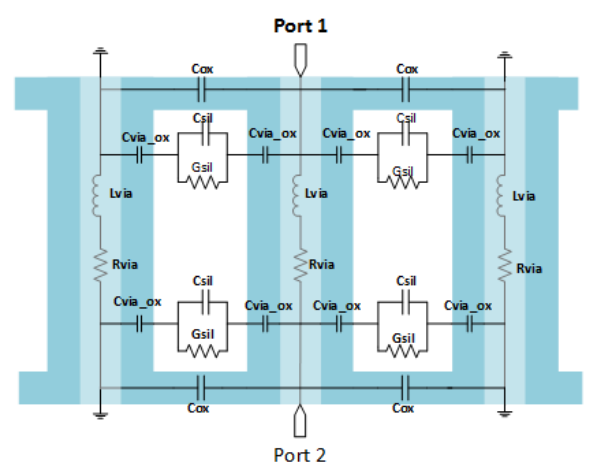

(a)

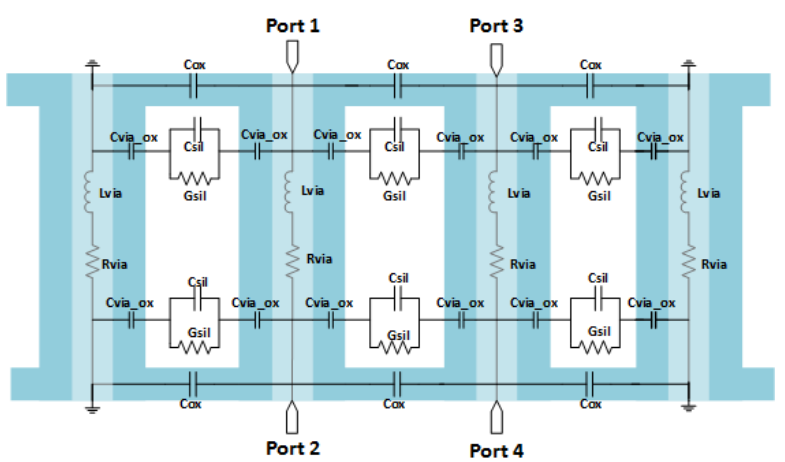

(b)

Figure 1. Equivalent Circuit Model of (a) a Standard GSG TSV and (b) a standard GSSG TSV [4]

\section{Proposed Oscillation Ring-BASEd 3D Testing SCHEMES}

Oscillation ring (OR) test is a useful and efficient method to detect faults in functional circuits and devices [5-6]. An oscillation ring is a closed loop with an odd number of signal inversions. Intuitively, if an oscillation ring fails to oscillate, it implies that there exists stuck-at or open faults in the components of the oscillation ring. The period of the oscillation signal can also be measured to test the delay faults. For example; in a circuit with gate or path delay faults, the oscillation frequency is different from the fault-free circuit. By observing the oscillation signal at the circuit output, one can decide whether the circuit-under-test is faulty or not. To evaluate the impact of a TSV on 3D circuit performance due to the additional delay induced by a TSV, an Oscillation Ring (OR) 3D Built-in Self-Test (BIST) testing schemes are designed and simulated using the Advanced Design System (ADS) and $0.25 \mu \mathrm{m}$ standard CMOS process. The target fault models of this testing architecture are TSV stuck-at, open, delay and slope degradation, and TSVs strong crosstalk coupling. If a triggered oscillator fails to oscillate, there exist stuck-at or open faults in the components of the RO. In addition, measuring the TSV delay fault can also be achieved. In this proposed testing scheme, a counter is included at the output of each triggered oscillator to detect TSVs delay faults.

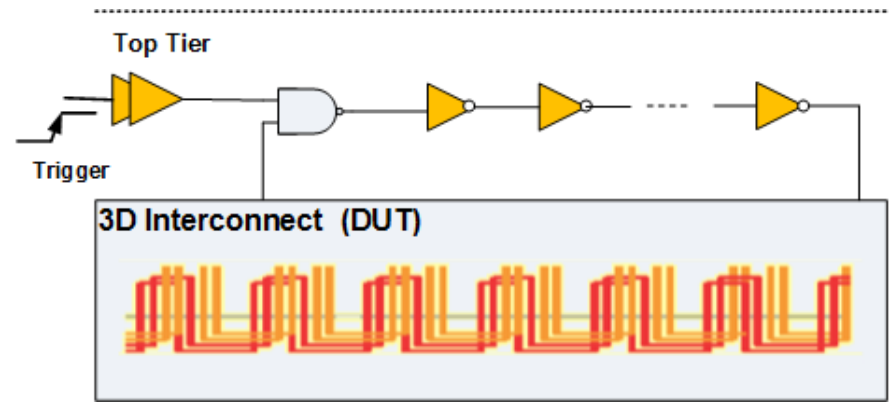

Figure 2. OR Testing Methodology for 3D Stacked ICs

\section{A. TSVs Test with Different Configurations}

In this testing scheme, a parallel ring oscillators (ROs) testing structure is presented, each RO has different routing TSVs configuration across different stacking tiers as shown in Figure 3. An edge detector circuitry is used to trigger ROs in parallel when the trigger enable signal (OR TEST) is asserted and a rising edge of the reference clock is detected. This will imply that each one will have a different oscillating frequency and thus a different counting value, which can be scanned out for off-chip testing. Since the frequency of each triggered oscillator is predetermined during the design phase, a delay fault can thus be detected and measured by inspecting the contents of the counters. In this test scheme, let assume that we have $\mathrm{m}$ triggered oscillators for different TSV configurations and the oscillation ring test is enabled by the OR_TEST signal. Also let assume that the triggered oscillator unloaded by TSVs is allowed to count only up to $\mathrm{n}$ at frequency of $\mathrm{f}_{0}$ and the counter contents of the other triggered oscillators, be $\mathrm{n}_{1}, \mathrm{n}_{2}, \ldots, \mathrm{n}_{\mathrm{m}}$, respectively. An estimation of the $i$ th ring's oscillation frequency $f_{i}$ can be approximated by:

$f_{i}=f_{0} * \frac{n i}{n}$

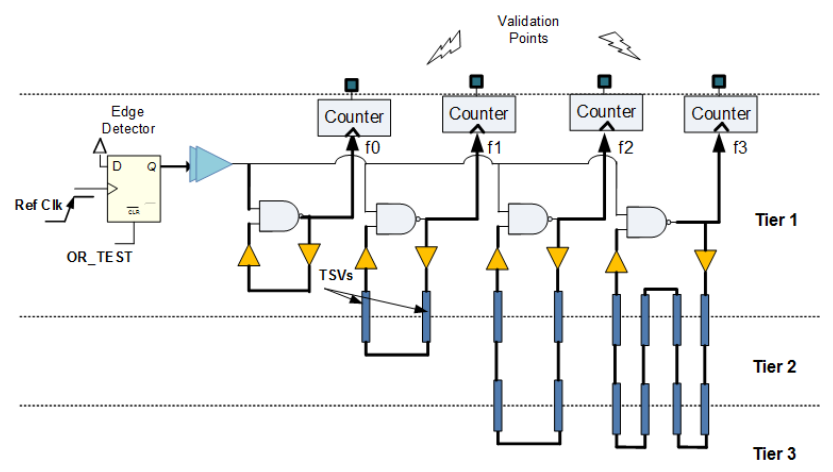

Figure 3. 3D Interconnect Test based on a Parallel triggered ROs Structure 
Figure 4 shows simulation results for the triggered oscillators connected with different $3 \mathrm{D}$ configurations based on the pimodel which is used to electrically characterize the TSV. Each triggered oscillator has different output frequencies where the triggered oscillator with unloaded TSV has the highest frequency $(1.37 \mathrm{GHz}$ ) compared to the ones loaded by TSVs. For example; the triggered oscillators loaded by 2 TSVs, 4 TSVs, and 8 TSVs have the following frequencies: $662 \mathrm{MHz}$, $543 \mathrm{MHz}$, and $436 \mathrm{MHz}$ respectively. Each output frequency will have a different counter value. Figure 5 shows the counting values 'b111, 'b101, 'b010 for the 2 TSVs, 4 TSVs and 8 TSVs respectively. Obviously, the addition of a TSV is similar to the addition of a short interconnect, thus leading to a degradation of rise time and more induced delay in the ring oscillator and thus slower oscillating frequency.

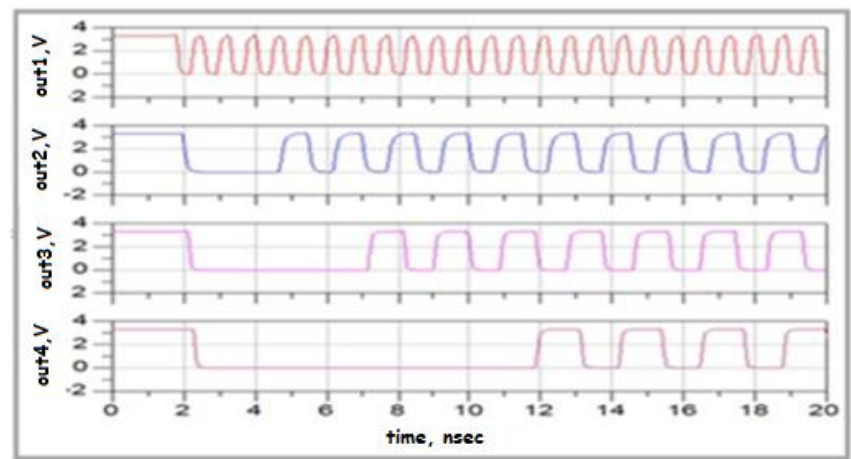

Figure 4. Output frequencies for the triggered oscillators

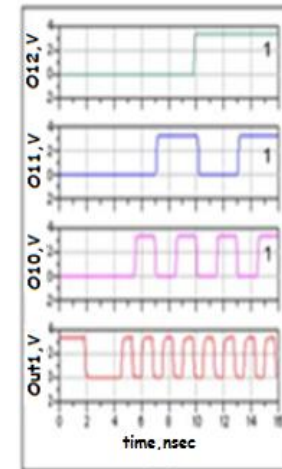

(a)

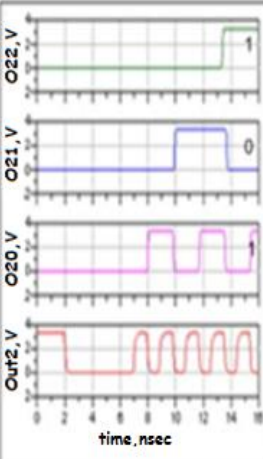

(b)

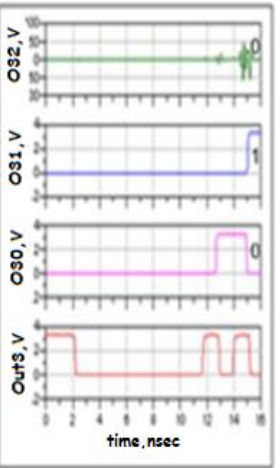

(c)
Figure 5 Output Frequencies and Counter Values for Different 3D Configuration (a) TSVs, (b) 4 TSVs, (c) 8 TSVs

\section{B. TSVs Rise Time Degradation Test}

To characterize the effect of the rise time degradation using the OR test, the capacitance of the thin oxide layer is changed from $10 \mathrm{fF}$ to $4 \mathrm{pF}$, which corresponds to $45 \mathrm{ps}$, and $1.3 \mathrm{~ns}$ rise time respectively as shown in Figure 6. This will presents the importance of RC delay caused by the time needed to load TSV oxide capacitance. Figure 7 shows the output frequency and the counter values for triggered oscillators loaded by 2 TSVs for both cases ( $45 \mathrm{ps}, 1.3 \mathrm{~ns}$ ). The $1.15 \mathrm{Ghz}$ output frequency with counter value of ' $b 111$ corresponds to the $45 \mathrm{ps}$ TSV rise time, and the $833 \mathrm{MHz}$ output frequency with counter value 'b010 corresponds to the $1.3 \mathrm{~ns}$ TSV rise time.

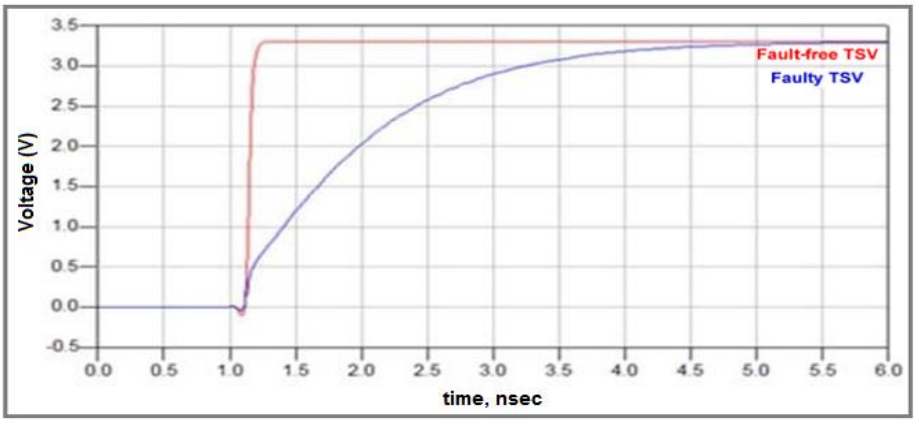

Figure 6. TSV Rise Time (red: 45ps, blue: $1.3 \mathrm{~ns}$ )
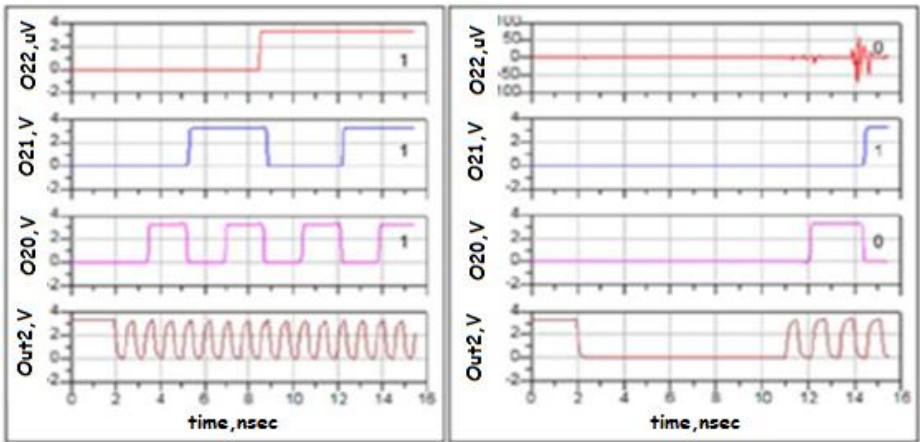

Figure 7. Output Frequencies and Counter Values for (a) $45 \mathrm{ps}$ TSV rise time, (b) $1.3 \mathrm{~ns} \mathrm{TSV}$ rise time

\section{TSVs Crosstalk Test}

In this test, a consecutive triggered parallel ROs structure running same frequency is used to characterize the crosstalk effect between TSVs. Figure 8 shows four triggered oscillators; two oscillators are crosstalk-coupled and the other two are crosstalk-free. The proposed ROs parallel structure creates a delta phase shift difference between each consecutive triggered oscillators, which is equal to the time difference between the delay buffer chain and the oscillation time period of the triggered oscillators. 3D crosstalk detection can be achieved by observing the frequency of crosstalk-coupled oscillators, which is different from the frequency of the crosstalk-free oscillators.

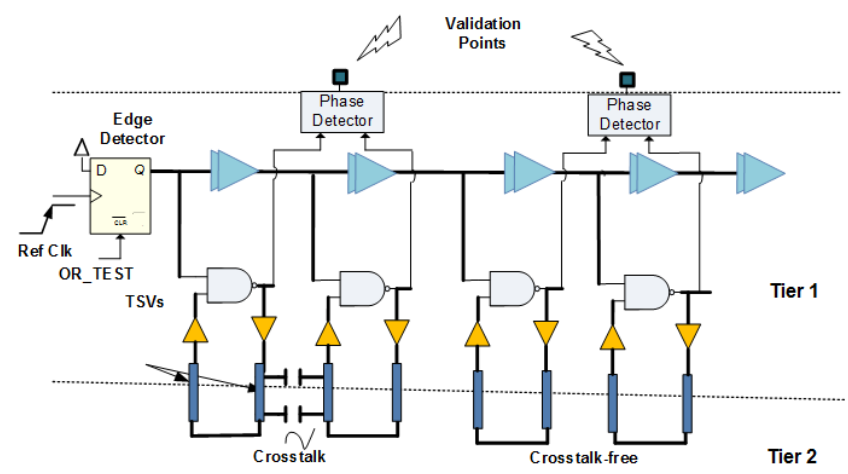

Figure 8. 3D Crosstalk Detection Scheme

Figure 9 shows the output frequency for both crosstalkcoupled $(725 \mathrm{MHz})$ and crosstalk-free oscillators $(667 \mathrm{MHz})$. 


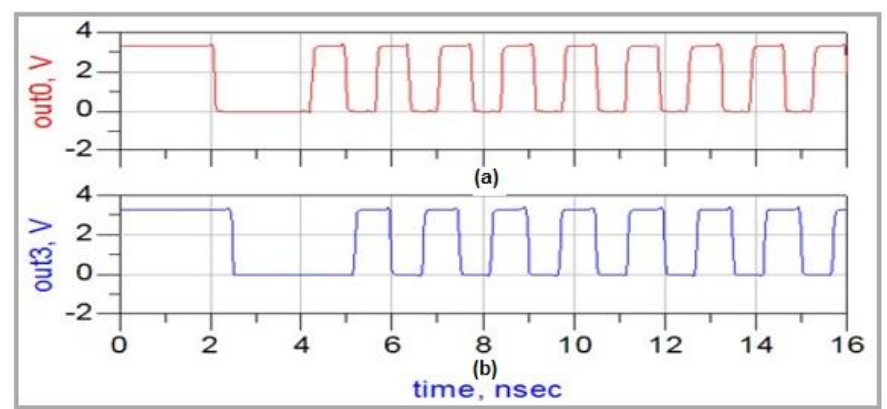

Figure 9. Output frequency for (a) Crosstalk-coupled and (b) Crosstalk-free oscillators

Interestingly, the two crosstalk-coupled triggered oscillators have less oscillation time delay (i.e. faster frequency) than the crosstalk-free oscillators, and the phase difference between the two crosstalk-coupled oscillators diminishes due to the coupling effect as shown in Figure 10. Phase detection at the output of the crosstalk-coupled triggered oscillators can be used as an indicator for strong coupling between TSVs. On the other hand, the edges of crosstalk-free oscillators are still separated by a deterministic phase shift dictated by the time difference between the delay buffer chain and the ring oscillation time period.

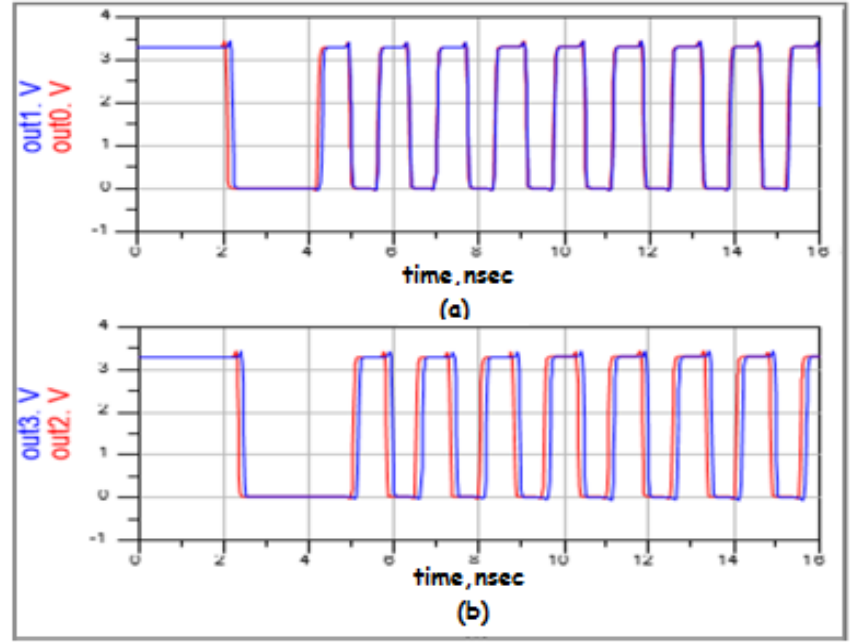

Figure 10. Simulated Results for 3D Interconnect Crosstalk (a) Crosstalk-coupled (b) Crosstalk free

\section{CONCLUSION}

In this work, an oscillation ring (OR) 3D testing methodology to detect and identify faulty TSVs and characterize their impact on 3D circuits performance is presented. The proposed OR testing methodology adopt a parallel ROs structure, which is dedicated to detect TSV faults such as stuckat, open, delay faults and crosstalk coupling between TSVs. The parallel RO structure can be built in different 3D configurations connected through TSVs across different 3D stacking tiers to evaluate and characterize the reliability of TSVs. For open and short faults, the circuit outputs of this test scheme are based on pass/fail criteria. TSVs delay and rise time degradation can be detected based on the counter data for each triggered RO. In addition, the proposed testing structure provides a new way of detecting a strong noise coupling between TSVs. In this test, the outputs of the coupled ring oscillators, which is running at same frequency, will eventually lock in phase due to the coupling noise between TSVs, where a phase detection circuit can be used to indicate a strong TSVs crosstalk noise.

\section{REFERENCES}

[1] D. M. Jang, C. Ryu, K. Y. Lee, B. H. Cho, J. Kim, T. S. Oh, W. J. Lee, and $\mathrm{J} . \mathrm{Yu}$, "Development and evaluation of 3-D SiP with vertically interconnected through silicon vias (TSV)," Proc 2007 Elect. Comp. and Tech. Conf, pp. 847-852, 2007.

[2] C. Ryu, D. Chung, J. Lee, K. Lee, T. Oh and J. Kim, "High frequency electrical circuit model of chip-to-chip vertical via interconnection for 3D chip stacking package," IEEE 14th Topical Meeting on Electrical Performance of Electronic Packaging, pp. 151-154, 2005.

[3] Z. Xu, A. Beece, K. Rose, T. Zhang, and J. Q. Lu, "Modeling and evaluation for electrical characteristics of Through Strata Vias (TSVs) in three-dimensional integration," IEEE 3D System Integration Conference, 2009.

[4] C. Ryu, J. Lee, H. Lee, K. Lee, T. Oh, and J. Kim, "High frequency electrical model of through wafer via for 3-D stacked chip packaging," Electronics System integration Technology Conference, vol. 1, pp. 215220, 2006.

[5] M. Kaneko, and K. Sakaguchi, "Oscillation fault diagnosis for analog circuits based on boundary search with perturbation model," IEEE ISCAS, pp. 93-96, 1994

[6] K. Arabi, and B. Kamiska, "Oscillation built-in self test (OBIST) scheme for functional and structural testing of analog and mixed-signal integrated circuits," Int'l Test Conf. pp. 786-795, 1997. 